\title{
Ferulic acid modulates nitric oxide synthase expression in focal cerebral ischemia
}

\author{
Phil-Ok Koh* \\ Department of Anatomy, College of Veterinary Medicine, Research Institute of Life Science, \\ Gyeongsang National University, Jinju, Korea
}

\begin{abstract}
Nitric oxide (NO) is generated by three different NO synthase (NOS) isoforms, endothelial NOS (eNOS), inducible NOS (iNOS), and neuronal NOS (nNOS). It is known that eNOS produces NO, which exerts a protective effect, while iNOS produces NO with neurotoxic effects. Ferulic acid preserves neuronal cells against from cerebral ischemia and glutamate-induced excitotoxicity. This study confirmed the neuroprotective effect of ferulic acid and investigated the levels of three NOS isoforms in focal cerebral ischemia with or without ferulic acid. Rats were immediately treated with ferulic acid (100 $\mathrm{mg} / \mathrm{kg}$, i.v.) after middle cerebral artery occlusion (MCAO). Brains tissues were collected at $24 \mathrm{~h}$ after the onset of occlusion. The expressions of these three isoforms in cerebral ischemia with ferulic acid were analyzed using Western blot technique. Ferulic acid treatment significantly decreases the number of TUNEL-positive cells in the cerebral cortex against MCAO injury. The levels of eNOS decreased in MCAO-operated animals, while ferulic acid treatment attenuated the MCAO-induced decrease of eNOS. However, iNOS and nNOS expression levels increased during MCAO, and ferulic acid prevented injury-induced increase of these isoforms. Thus, these findings suggest that the up- and down modulation of three isoforms by ferulic acid is associated with a neuroprotective mechanism.
\end{abstract}

Key words: Ferulic acid, neuroprotection, NOS

Received 25 August 2012; Revised version received 13 October 2012; Accepted 9 November 2012

Ferulic acid (4-hydroxy-3-methoxycinnamic acid, FA) is well known as an effective component of Chinese medicinal herbs, such as Angelica sinensis, Cimicifuga heracleifolia and Lignsticum chuangxiong. These components have been used as traditional therapy agents for stroke. Ferulic acid has antioxidant and anti-aging proprieties, exerts beneficial effect against various diseases [1,2]. Ferulic acid reduces cerebral ischemic injury by modulating inflammatory reaction and regulating of various signaling pathway $[1,3,4]$. Ferulic acid also inhibits neuronal cells death from glutamateinduced excitotoxity in cultured cortical neurons [4].

Nitric oxide (NO) acts as an important mediator of numerous processes in the nervous, cardiovascular, and immune systems $[5,6]$. Nitric oxide (NO) plays essential functions for the physiological and pathological processes in the central nervous system [5,6]. Moreover, NO has an effect on both neuroprotective and neurotoxic in focal cerebral ischemia, and mediates pathophysiologic states as diverse as stroke, neurodegenerative diseases, and hypertension [7-9]. Nitric oxide (NO) is synthesized from L-arginine by the action of nitric oxide synthase (NOS). NOS has three isoforms including inducible NOS (iNOS), endothelial NOS (eNOS), and neuronal NOS (nNOS). The inducible isoform is calciumindependent and is expressed in response to various inflammatory stimuli, and thus, it is accepted that iNOS produces NO with neurotoxic effects [10-12]. The neuronal and endothelial isoform are calcium-dependent and $\mathrm{NOS}$ generates $\mathrm{NO}$ which exerts a protective effect

\footnotetext{
*Corresponding author: Phil-Ok Koh, Department of Anatomy, College of Veterinary Medicine, Gyeongsang National University, 900 Gajwa-dong, Jinju, Gyeongnam 660-701, Korea Tel: +82-55-772-2354; Fax: +82-55-772-2349; E-mail: pokoh@gnu.ac.kr
}

This is an Open Access article distributed under the terms of the Creative Commons Attribution Non-Commercial License (http://creativecommons.org/licenses/ by-nc/3.0) which permits unrestricted non-commercial use, distribution, and reproduction in any medium, provided the original work is properly cited. 
[13].

NOS is involved in the mechanisms of cerebral ischemic injury $[14,15]$. A previous study demonstrated that eNOS knockout mice exhibit an enlarged infarct, and NO generated from eNOS has a beneficial effect through preservation of the cerebral blood flow and anti-platelet aggregation in cerebral ischemic injury [16,17]. Moreover, inhibition of iNOS improves neurological deficits, reduces infarct volume after cerebral ischemia, and reduces susceptibility to focal cerebral ischemia $[14,15]$. Although previous studies have demonstrated the neuroprotective mechanisms of ferulic acid, little data is available regarding the levels of NOS isoforms expression. Thus, this study examined whether ferulic acid mediates the expression levels of NOS isoforms in focal cerebral ischemia.

Male Sprague-Dawley rats (220-230 g) were purchased from Samtako (Animal Breeding Center, Osan, Korea). All animal experiments were followed a protocol approved by the Committee for Animal Experimentation at the Gyeongsang National University (GNU-120806R0034). Rats were divided into four groups: vehicle+ sham group, ferulic acid+sham group, vehicle+middle cerebral artery occlusion (MCAO) group, and ferulic acid+MCAO group ( $n=10$ per group). Ferulic acid (100 $\mathrm{mg} / \mathrm{kg}$, Sigma, St. Louis, MO, USA) was injected as previously described method [1]. Ferulic acid was dissolved in normal saline and injected intravenously immediately after middle cerebral artery occlusion (MCAO). Vehicle was given the same volume of normal saline.

MCAO was operated as previously described method [18]. Rats were anesthetized with sodium pentobarbital $(100 \mathrm{mg} / \mathrm{kg})$. Briefly, the right common carotid artery, external carotid artery, and internal carotid artery were exposed through a midline cervical incision. A nylon filament (4/0) with blunted tip was inserted through the right internal carotid artery to the base of the middle cerebral artery, thereby occluding the origin of the MCA. The sham operated animals were subjected to the same procedures except the insertion of filament. At $24 \mathrm{~h}$ after the onset of permanent occlusion, animals were decapitated, and the brains were rapidly removed.

Brains were fixed with $4 \%$ neutral buffer paraformaldehyde solution and embedded with paraffin and sectioned for TUNEL staining. TUNEL immunohistochemistry was carried out using the DNA Fragmentation Detection Kit (Oncogene Research Products, Cambridge,
MA, USA). Briefly, sections were incubated with proteinase $\mathrm{K}(20 \mu \mathrm{g} / \mathrm{mL})$ for $5 \mathrm{~min}$, rinsed with PBS and incubated with terminal deoxynucleotidyl transferase (TdT) equilibration buffer for $30 \mathrm{~min}$. They were incubated with a mixture of $\mathrm{TdT}$ and digoxigenin-labelled dideoxy nucleotide in a humidified chamber at $37^{\circ} \mathrm{C}$ for $1 \mathrm{~h}$. After addition of the stop solution and blocking buffer, sections were incubated with an anti-digoxigenin antibody conjugated with horseradish peroxidase for $1 \mathrm{~h}$. After sections were washed in PBS, the color reaction was performed with DAB (Sigma, St. Louis, MO, USA) solution with $0.03 \%$ hydrogen peroxide for $3 \mathrm{~min}$. Sections were counterstained with hematoxylin. TUNELpositive cells were quantified using light microscopy. The percentage of TUNEL-positive cells is described as the percentage of the number of TUNEL-positive cells to the total number of cells in cerebral cortex field.

For Western blot analysis, cerebral cortex tissues were quickly lysed in buffer [1\% Triton X-100, 1 mM EDTA in $1 \times \mathrm{PBS}(\mathrm{pH} 7.4)]$ containing $10 \mu \mathrm{M}$ leupeptin and 200 $\mu \mathrm{M}$ phenylmethylsulfonyl fluoride. The lysates were centrifuged at $15,000 \mathrm{~g}$ for $20 \mathrm{~min}$ at $4^{\circ} \mathrm{C}$. The supernatants were collected and the protein concentration was measured using the bicinchoninic acid (BCA) kit (Pierce, Rockford, IL, USA) according to the manufacturer's protocol. Thirty micrograms of total protein was boiled at $100^{\circ} \mathrm{C}$ in sodium dodecyl sulfate (SDS) gel loading buffer for $10 \mathrm{~min}$ and applied to each lane on to $10 \%$ SDS polyacrylamide gels (SDS-PAGE). After electrophoresis, separated proteins were electrotransferred to the polyvinylidene fluoride (PVDF) membranes (Millipore, Billerica, MA, USA) in transfer buffer. The membrane was incubated in $5 \%$ skim milk containing $0.1 \%$ Tween 20 for $60 \mathrm{~min}$ to block nonspecific binding, then incubated with the following antibodies: anti-eNOS, anti-iNOS, anti-nNOS, and anti-actin (diluted 1:1000, Santa Cruz Biotechnology, Santa Cruz, CA, USA) as primary antibody. After washing, the membrane was incubated with anti-rabbit HRP-linked IgG (1:5000, Pierce, Rockford, IL, USA), and the ECL Western blot analysis system (Amersham Pharmacia Biotech, Piscataway, NJ, USA) according to the manufacturer's protocol was used for detection. The densitometric analysis intensity analysis was carried out using SigmaGel 1.0 (Jandel Scientific, San Rafael, CA, USA) and SigmaPlot 4.0 (SPSS Inc., Point Richmond, CA, USA). All data are expressed as mean \pm S.E.M. The results in each group were compared by one-way analysis of 

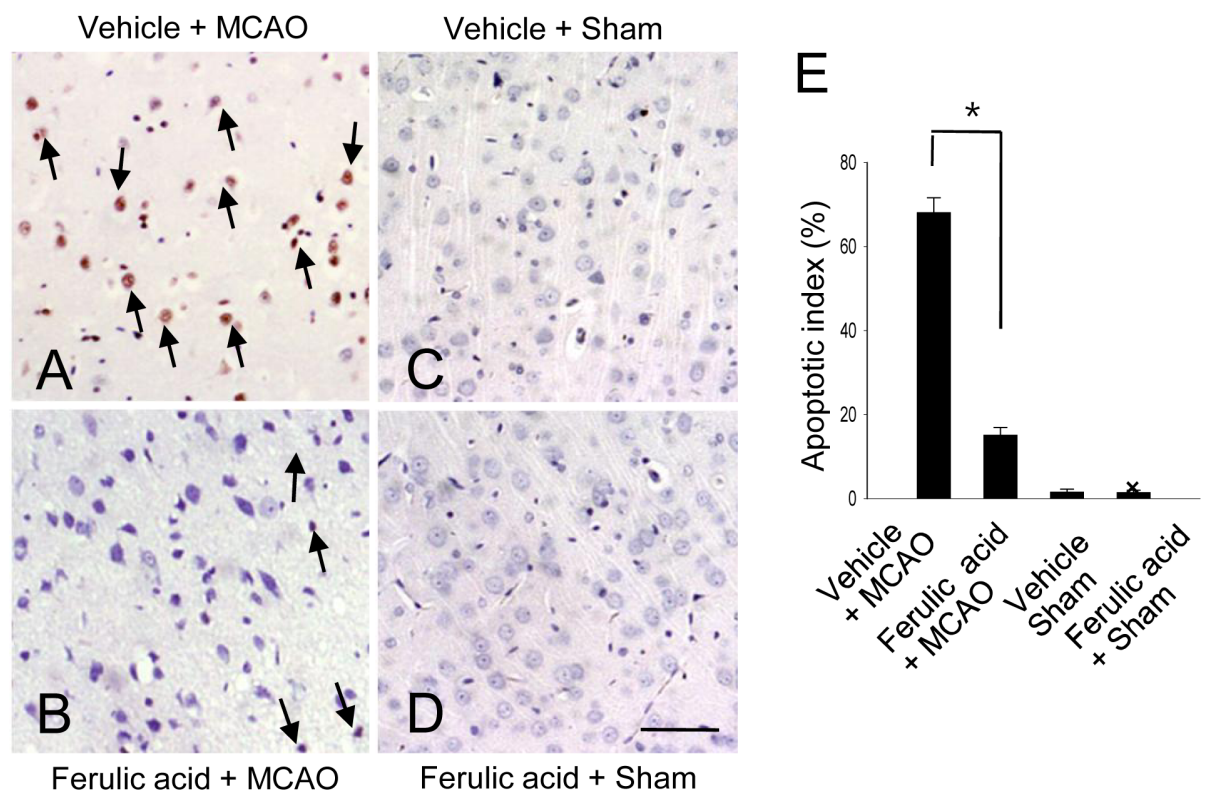

Figure 1. Representative photos of TUNEL staining in the cerebral cortex from vehicle+MCAO (A), ferulic acid+MCAO (B), vehicle+sham (C), ferulic acid+sham (D) animals. Ferulic acid treatment attenuates the number of TUNEL-positive cells in MCAOoperated animals. Arrows indicate the positive cells of TUNEL staining. Scale bar $=100 \mu \mathrm{m}$. Apoptotic index indicate the percentage of TUNEL-positive cells in the ischemic regions $(E)$. Data $(n=5)$ are represented as mean \pm S.E.M. ${ }^{*} P<0.01$.

variance (ANOVA) followed by Student's $t$ test. The difference for comparison was considered significant at $* P<0.05$.

TUNEL staining confirmed the neuroprotective effects of ferulic acid against MCAO. The cerebral cortex of MCAO operated animals without ferulic acid treatment increased the number of TUNEL-positive cells compared to those of the sham-operated control (Figure 1A-D). However, ferulic acid treatment prevented the MCAOinduced increase in the number of TUNEL-positive cells. The apoptotic index was $70.5 \pm 2.3$ in the vehicle+ MCAO, whereas it was $17.6 \pm 2.5$ in the ferulic acid+ MCAO animals (Figure 1E).

Western blot analysis showed the expression levels of three NOS isoforms in MCAO operated animals with or without ferulic acid. MACO induced a decrease in eNOS levels, while ferulic acid treatment attenuated the injury-induced decrease of eNOS expression. The level of eNOS was $0.45 \pm 0.02$ and $0.82 \pm 0.02$ in the vehicle+ MCAO and ferulic acid+MCAO, respectively. In shamoperated animals, the levels of eNOS in vehicle- and ferulic acid-treated animals were $0.83 \pm 0.03$ and $0.84 \pm$ 0.01, respectively (Figure 2). In contrast to eNOS expression, MCAO leads to an increase in both iNOS and nNOS levels, while ferulic acid treatment attenuated

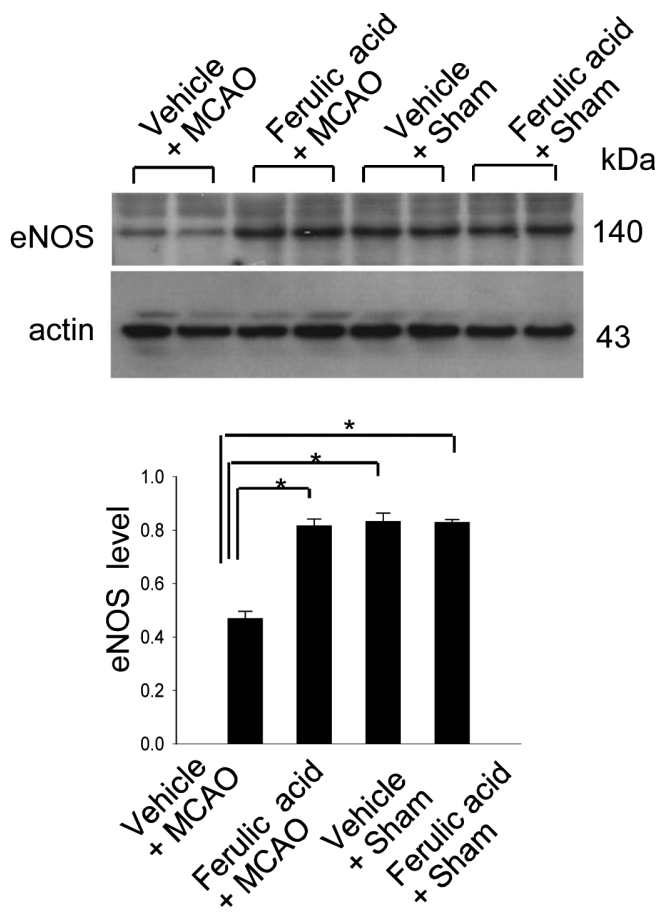

Figure 2. Western blot analysis of eNOS in the cerebral cortex from vehicle+MCAO, ferulic acid+MCAO, vehicle+sham, ferulic acid+sham animals. Each lane represents an individual experimental animal. Densitometric analysis is represented as a ratio, proteins intensity to actin intensity. Molecular weight $(\mathrm{kDa})$ are depicted at right. Data $(n=5)$ are represented as mean \pm S.E.M. ${ }^{*} P<0.05$. 


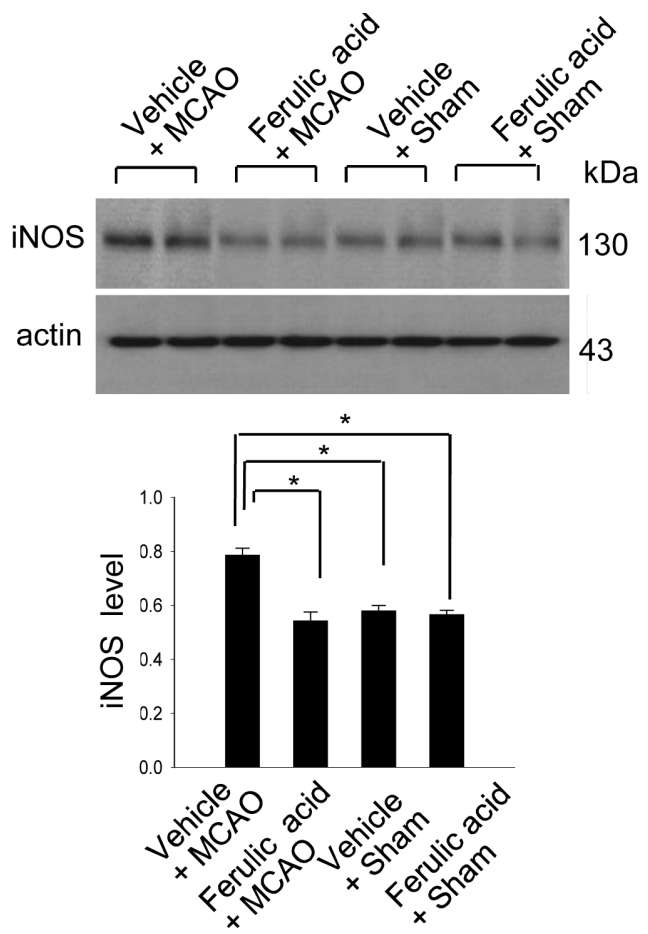

Figure 3. Western blot analysis of iNOS in the cerebral cortex from vehicle+MCAO, ferulic acid+MCAO, vehicle+sham, ferulic acid+sham animals. Each lane represents an individual experimental animal. Densitometric analysis is represented as a ratio, proteins intensity to actin intensity. Molecular weight $(\mathrm{kDa})$ are depicted at right. Data $(n=5)$ are represented as mean \pm S.E.M. ${ }^{\star} P<0.05$.

the MCAO-induced increase of these isoforms. The level of iNOS was $0.79 \pm 0.02$ in the vehicle $+\mathrm{MCAO}$, whereas it was $0.56 \pm 0.02$ in the ferulic acid $+\mathrm{MCAO}$ animals. In sham-operated animals, the level of iNOS was $0.58 \pm 0.01$ and $0.57 \pm 0.01$ in vehicle- and ferulic acid-treated animals, respectively (Figure 3). Moreover, the level of nNOS was $1.05 \pm 0.03$ and $0.94 \pm 0.02$ in the vehicle $+\mathrm{MCAO}$ and ferulic acid+MCAO, respectively, while the levels of nNOS in vehicle- and ferulic acidtreated animals during sham-operation were $0.89 \pm 0.02$ and $0.87 \pm 0.01$ (Figure 4).

Ferulic acid reduces infarct size and apoptotic process in focal cerebral ischemia by modulating of anti-oxidant and anti-inflammatory actions $[1,3]$. Our previous study also demonstrated that ferulic acid reduces infarct regions and preserves neuronal cell by modulating signaling pathways, including PI3K/Akt and caspase-3 activation, during focal cerebral ischemia [4]. In this study, the results of TUNEL staining clearly confirmed the neuroprotective effect of ferulic acid in focal cerebral ischemia. Ferulic acid attenuated MCAO-induced increases in the number of TUNEL staining positive cells.

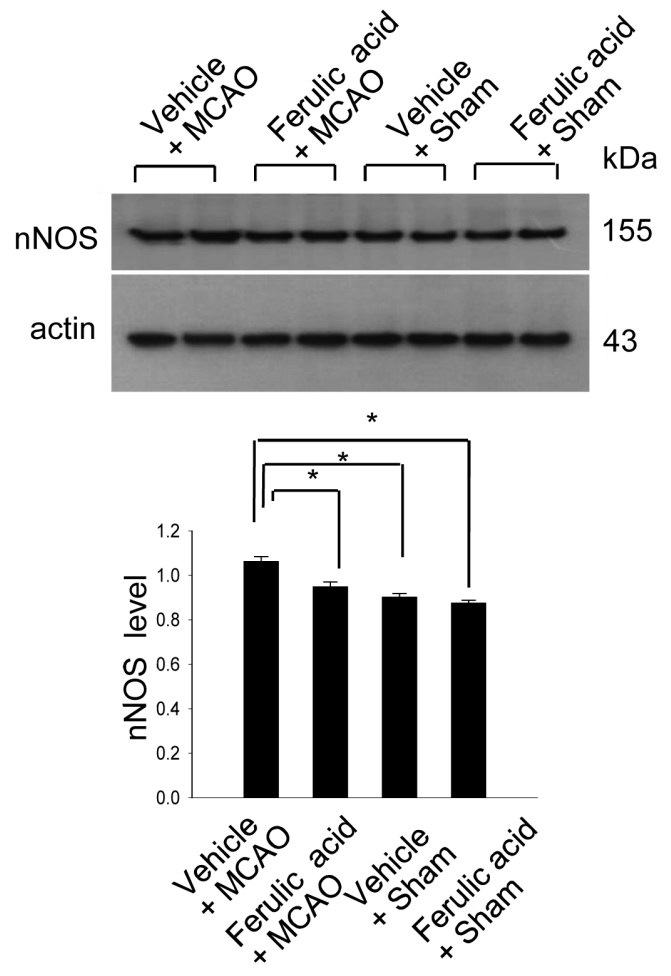

Figure 4. Western blot analysis of nNOS in the cerebral cortex from vehicle+MCAO, ferulic acid+MCAO, vehicle+sham, ferulic acid+sham animals. Each lane represents an individual experimental animal. Densitometric analysis is represented as a ratio, proteins intensity to actin intensity. Molecular weight $(\mathrm{kDa})$ are depicted at right. Data $(n=5)$ are represented as mean \pm S.E.M. ${ }^{*} P<0.05$.

NO, which is produced by eNOS, exerts an antiplatelet aggregation effect in cerebral ischemia and improves cerebral blood flow against ischemic condition $[16,17]$. eNOS, which is found in endothelial cells, is an important factor for endothelial function and performs an critical function for the pathophysiology of vascular endothelium $[19,20]$. Thus, the decline of eNOS expression induces extensive tissue damage by platelet aggregation and neutrophil infiltration [21]. In contrast, the rise of eNOS increases blood flow and mediates the protective mechanism of the endothelium [19]. Our result shows that eNOS levels decrease in MCAOoperated animals compared to sham-operated animals. This study confirms the fact that the decrease of eNOS expression leads to tissue damage [21]. Our data additionally demonstrated that eNOS expression levels decrease in MCAO-induced brain injury and ferulic acid treatment attenuates decreases of eNOS levels. Previous studies demonstrated the fact that ferulic acid exerts a beneficial pharmacological effects, which includes antiplatelet aggregation, anti-peroxidation, and anti-inflammatory 
reaction $[1,3,22]$. Thus, the maintenance of eNOS levels by ferulic acid treatment during MCAO injury mediates various functions, including anti-platelet aggregation and anti-peroxidation, leading to neuroprotective mechanisms.

Tissue hypoxia induces serious cell damage in focal cerebral ischemia. Ischemic condition accelerates the release of excitatory amino acids, nitric oxide, and free radicals, and induces microglial activation and programmed cell death [23-25]. iNOS is mainly expressed in astrocytes and activated microglia during focal brain ischemia [26]. iNOS knockout mice reduce brain damage after ischemia and the increased expression of iNOS contributes to neuronal injury [25]. In addition, NO by generated iNOS increases free radical formation and promotes brain damage after stroke [7]. This study shows that iNOS levels increase in MCAO-operated animals compared to sham-operated animals. The increases of iNOS levels are consistent with a previous report which showed that iNOS expression is increased after cerebral ischemia [14]. Moreover, ferulic acid treatment prevents the injury-induced increase of the iNOS levels. This study additionally demonstrated that ferulic acid regulates iNOS expression, and exerts a protective effect against iNOS-mediated oxidative stress in focal cerebral ischemia. Moreover, nNOS which exists in neuronal cells is expressed in similar pattern to iNOS expression. nNOS is increased in MCAO-operated animals compared to sham-operated animals. Ferulic acid treatment suppresses the up-regulation of nNOS in MCAO animals. It is known that nNOS plays prominent roles in neurodegeneration, and that the excessive production of $\mathrm{NO}$ from nNOS induces neurotoxicity [8]. This study demonstrated that ferulic acid suppresses the rise of nNOS that leads to neurotoxicity and cell damage, and exerts beneficial effects of neuroproction.

Ferulic acid attenuates the down-regulation of eNOS, and the rise of iNOS and nNOS in MCAO-induced brain injury. Taken together, these data demonstrate that ferulic acid contributes to a protective mechanism through NOS-mediated mechanisms in focal cerebral ischemia. Although further research is required to elucidate the mechanism by which ferulic acid regulates NO system after focal cerebral ischemia, these findings provide evidence that ferulic acid prevents neuronal cell death against focal cerebral ischemia, and that the up- and down regulation of three NOS isoforms by ferulic acid may mediate the protective mechanism of ferulic acid.

\section{Acknowledgments}

This research was supported by the National Research Foundation of Korea (NRF) grant funded by the Korea government (MEST) (No.2010-0007881). I really thank Ms. J.H. Sung for substantial technical help.

\section{References}

1. Cheng CY, Ho TY, Lee EJ, Su SY, Tang NY, Hsieh CL. Ferulic acid reduces cerebral infarct through its antioxidative and antiinflammatory effects following transient focal cerebral ischemia in rats. Am J Chin Med 2008; 36(6): 1105-1119.

2. Manosroi A, Chutoprapat R, Abe M, Manosroi W, Manosroi J. Anti-aging efficacy of topical formulations containing niosomes entrapped with rice bran bioactive compounds. Pharm Biol 2012; 50(2): 208-224.

3. Cheng CY, Su SY, Tang NY, Ho TY, Chiang SY, Hsieh CL. Ferulic acid provides neuroprotection against oxidative stressrelated apoptosis after cerebral ischemia/reperfusion injury by inhibiting ICAM-1 mRNA expression in rats. Brain Res 2008; 1209: 136-150

4. Koh PO. Ferulic acid prevents the cerebral ischemic injuryinduced decrease of Akt and Bad phosphorylation. Neurosci Lett 2012; 507(2): 156-160.

5. Szabo C. Physiological and pathophysiological roles of nitric oxide in the central nervous system. Brain Res Bull 1996; 41(3): 131-141.

6. Yun HY, Dawson VL, Dawson TM. Neurobiology of nitric oxide. Crit Rev Neurobiol 1996; 10(3-4): 291-316.

7. Dalkara T, Moskowitz MA. The complex role of nitric oxide in the pathophysiology of focal cerebral ischemia. Brain Pathol 1994; 4(1): 49-57.

8. Samdani AF, Dawson TM, Dawson VL. Nitric oxide synthase in models of focal ischemia. Stroke 1997; 28(6): 1283-1288.

9. Davies SA, Stewart EJ, Huesmann GR, Skaer NJ, Maddrell SH, Tublitz NJ, Dow JA. Neuropeptide stimulation of the nitric oxide signaling pathway in Drosophila melanogaster Malpighian tubules. Am J Physiol 1997; 273(2): 823-827.

10. Vane JR, Mitchell JA, Appleton I, Tomlinson A, Bishop-Bailey D, Croxtall J, Willoughby DA. Inducible isoforms of cyclooxygenase and nitric-oxide synthase in inflammation. Proc Natl Acad Sci U S A 1994; 91(6): 2046-2050.

11. Galea E, Reis DJ, Xu H, Feinstein DL. Transient expression of calcium-independent nitric oxide synthase in blood vessels during brain development. FASEB J 1995; 9(15): 1632-1637.

12. Sparrow JR. Inducible nitric oxide synthase in the central nervous system. J Mol Neurosci 1994-1995; 5(4): 219-229.

13. Hashiguchi A, Yano S, Morioka M, Hamada J, Ushio Y, Takeuchi Y, Fukunaga K. Up-regulation of endothelial nitric oxide synthase via phosphatidylinositol 3-kinase pathway contributes to ischemic tolerance in the CA1 subfield of gerbil hippocampus. J Cereb Blood Flow Metab 2004; 24(3): 271-279.

14. Iadecola C, Zhang F, Casey R, Clark HB, Ross ME. Inducible nitric oxide synthase gene expression in vascular cells after transient focal cerebral ischemia. Stroke 1996; 27(8): 1373-1380.

15. Iadecola C, Zhang F, Casey R, Nagayama M, Ross ME. Delayed reduction of ischemic brain injury and neurological deficits in mice lacking the inducible nitric oxide synthase gene. J Neurosci 1997; 17(23): 9157-9164.

16. Huang Z, Huang PL, Ma J, Meng W, Ayata C, Fishman MC, Moskowitz MA. Enlarged infarcts in endothelial nitric oxide synthase knockout mice are attenuated by nitro-L-arginine. J Cereb Blood Flow Metab 1996; 16(5): 981-987. 
17. Iadecola C, Pelligrino DA, Moskowitz MA, Lassen NA. Nitric oxide synthase inhibition and cerebrovascular regulation. J Cereb Blood Flow Metab 1994; 14(2): 175-192.

18. Longa EZ, Weinstein PR, Carlson S, Cummins R. Reversible middle cerebral artery occlusion without craniectomy in rats. Stroke 1989; 20(1): 84-91.

19. Albrecht EW, Stegeman CA, Heeringa P, Henning RH, van Goor $\mathrm{H}$. Protective role of endothelial nitric oxide synthase. J Pathol 2003; 199(1): 8-17.

20. Marletta MA. Nitric oxide synthase: aspects concerning structure and catalysis. Cell 1994; 78(6): 927-930.

21. Heeringa P, Steenbergen E, van Goor H. A protective role for endothelial nitric oxide synthase in glomerulonephritis. Kidney Int 2002; 61(3): 822-825.

22. Dong TT, Zhao KJ, Gao QT, Ji ZN, Zhu TT, Li J, Duan R, Cheung AW, Tsim KW. Chemical and biological assessment of a chinese herbal decoction containing Radix Astragali and Radix Angelicae Sinensis: Determination of drug ratio in having optimized properties. J Agric Food Chem 2006; 54(7): 2767-2774.

23. Hallenbeck JM, Dutka AJ. Background review and current concepts of reperfusion injury. Arch Neurol 1990; 47(11): 12451254.

24. Aronowski J, Strong R, Grotta JC. Reperfusion injury: demonstration of brain damage produced by reperfusion after transient focal ischemia in rats. J Cereb Blood Flow Metab 1997; 17(10): 1048-1056.

25. Kuroda S, Siesjo BK. Reperfusion damage following focal ischemia: pathophysiology and therapeutic windows. Clin Neurosci 1997; 4(4): 199212.

26. Jander S, Schroeter M, Stoll G. Role of NMDA receptor signaling in the regulation of inflammatory gene expression after focal brain ischemia. J Neuroimmunol 2000; 109(2): 181-187. 